\title{
A 19 Year-Old Man With Chest Pain
}

\author{
Sam Barasch, MS IV, Sugeet Jagpal, MD
}

\section{Case Report}

A 19-year-old man with no past medical history presented to the ER with a sore throat, cough, and pleuritic chest pain. The patient had been well until 1 month before admission, when he developed a sore throat and felt ill. He presented to the emergency department twice for these symptoms. On the first visit, the patient had a positive rapid strep test. He was diagnosed with strep throat and treated with penicillin IM. However, his sore throat persisted. Five days before admission, the patient developed a non-productive cough. One day prior to admission he developed severe right sided chest pain that was throbbing, pleuritic, and radiated to his right shoulder. The pain, which was initially relieved by sitting forward and with Ibuprofen, continued to worsen, prompting the patient's mother to bring him back to be re-evaluated.

Upon presentation, the patient denied shortness of breath, fevers, or chills. He had no personal history of prenatal or childhood disease, and denied family history of early heart disease, lung disease, cancer or bleeding disorders. He denied tobacco, alcohol, or illicit drug use. He reported being heterosexual with 4-5 lifetime sexual partners, and one new partner in the prior two months.

On examination, the patient was a well nourished, well developed, young African American man in moderate distress. The blood pressure was $117 / 57$, heart rate 66 , respiratory rate 16 , oxygen saturation $98 \%$ on 2 L nasal cannula and temperature $37.5^{\circ} \mathrm{C}$. The physical exam was remarkable only for slight pharyngeal erythema and enlarged, non-purulent tonsils. His heart was without murmurs, rubs, or gallops. His lungs were clear to auscultation bilaterally. Initial labwork was unremarkable, with a normal white blood cell count. A chest radiograph revealed findings in the right lower lobe concerning for subsegmental atelectasis versus consolidation. Electrocardiogram demonstrated an incomplete right bundle branch block. A thoracic CT demonstrated a large right lower lobe pulmonary embolism with an associated area of pulmonary infarction.

Given the finding of the patient's pulmonary embolism, our top three differential diagnoses were a primary underlying hypercoagulable state, an infection predisposing the patient to a hypercoaguable state, or an underlying malignancy. The patient was admitted to the hospital, blood work was drawn, and he was started on Warfarin with an Enoxaparin bridge. During his work up, the patient was found to have elevated antistreptolysin O titers, consistent with a prior streptococcus infection. Blood cultures were negative. In addition, HIV, HSV, gonorrhea, chlamydia, group A strep, and H1N1 PCR were all negative. $\mathrm{CT}$ of the abdomen and pelvis and testicular ultrasound were normal, without any signs of malignancy. A rheumatologic workup, including an anti-nuclear antibody and anti-neutrophil cytoplasmic antibody were negative. A hypercoagulable work up was sent prior to initiation of anticoagulation therapy. Protein C, protein S, factor V leiden, antithrombin III, and anticardiolipin antibodies were normal. Eventually, after the patient had been discharged, his lupus anticoagulant levels returned as abnormally elevated. The patient was diagnosed with primary antiphospholipid syndrome by his primary care doctor and was informed he would require lifelong anticoagulation.

\section{Discussion}

Primary antiphospholipid syndrome is an auto-immune disease typically discovered when a patient has vascular thromboses or pregnancy complications in the presence of elevated anticardiolipin antibodies or lupus anticoagulant antibodies. Primary antiphospholipid syndrome can be diagnosed in the absence of broader auto-reactive antibody production, i.e. lupus. Detection of the auto-immune antibodies must be found on two separate occasions more than six weeks apart.

Of the laboratory criteria, anticardiolipin antibodies are more sensitive while the lupus anticoagulant antibodies are more specific. Lupus anticoagulant antibodies were originally found in lupus patients who had a prolonged activated partial thromboplastin time (aPTT). While patients with the lupus anticoagulant had prolonged in vitro coagulation studies, in vivo they were hyper-coaguable. Thus, these patients had high PTT or INR on blood work, in combination with a paradoxical tendency to clot in their body. This paradox is due to the fact that, in vitro, the lupus anticoagulant antibody binds to critical phopholipids and prevents them from activating factor $\mathrm{X}$ and prothrombin. In vivo, however, the antiphospholipid antibody binds to a multitude of targets that result in a hyper-coaguable state.

The lupus anticoagulant antibody has many significant targets within the body. The most clinically important in vivo target is $\beta_{2}$-glycoprotien. ${ }^{2}$ Many patients have circulating lupus anticoagulant antibodies that target phospholipids only, and do not have clinically significant thrombosis. However, when the antibodies target both phospholipids and $\beta_{2}$-glycoprotien I, the patients become much more likely to have a thrombus that causes symptoms. Antiphospholipid antibodies also promote coagulation by enhancing activation and aggregation of platelets, activation and expression of adhesion molecules on endothelium, and activation of the coagulation cascade.

Infection can be an inciting factor for a thrombotic event in a patient with a circulating, but clinically silent, antiphospholipid antibody. Many different pathogens are cited, including HIV, HCV, VZV, H influenza, Streptococcus species, and Staphylococcus aureus. Common systems infected prior to 
thrombosis include skin, pulmonary, urinary, and upper respiratory tract. ${ }^{4}$ Some investigators propose a "two hit" hypothesis in which patients with underlying antiphospholipid antibodies receive a second hit when a pathogenic epitope is a molecular mimic of $\beta_{2}$-glycoprotein I or when an inflammatory cascade is activated by toll-like receptors on phagocytes of the innate immune system. It is possible this happened to our patient: his previously silent circulating antibodies were activated by an upper respiratory streptococcus infection, causing an inflammatory cascade that resulted in a thrombotic event.

Treatment for patients with primary antiphospholipid syndrome centers on anticoagulation therapy. An INR of 2-3 (maintained by oral warfarin) has been shown to reduce the probability of recurrent thrombosis. In catastrophic cases where life threatening thromboses affect multiple organs and cause microvascular disease, treatment includes high dose steroids, intravenous immunoglobulin, cyclophosphamide, or plasmapharesis. Treatment in a hemodynamically compromised patient with pulmonary embolism can also include embolectomy. For prevention of late term pregnancy loss related to primary anti-phospholipid syndrome, low dose aspirin and subcutaneous prophylactic heparin have been shown to prevent complications. For patients refractory to anti-coagulation, long term immunomodulating agents should be added.
Our patient did not have catastrophic antiphospholipid syndrome, and is currently doing well on oral anticoagulation.

\section{Take home points:}

1) Primary antiphospholipid syndrome is vascular thromboses or pregnancy complications and anticardiolipin antibodies or lupus anticoagulant antibodies.

2) Anticardiolipin antibodies are more sensitive while lupus anticoagulant is more specific for diagnosing antiphospholipid syndrome.

3) Infection can prompt a circulating but clinically silent antiphospholipid antibody to cause a thrombotic event. Exact mechanism of this is unknown.

\section{References}

1. Lichtman, Beutler, Kipps, Seligsohn, Kaushansky, Prchal. Williams Hematology. 7th edition, Ch. 123 "The Antiphospholipid Syndrome: Overview."

2. Levine, Branch, Rauch. "The Antiphospholipid Syndrome." The New England Journal of Medicine. March 2002, Vol 346:10: 752-763.

3. Cervera, Asherton, Acevedo, Gomez-Puerta, Espinosa, de la Red, Gil, Ramos-Casals, Garcia-Carrasco, Ingelmo, Font. "Antiphospholipid Syndrome associated with infections: clinical and microbiological characteristics of 100 patients." Annals of the Rheumatic Diseases. 2004: 63: 1312-1317.

4. Shoenfeld, Blank, Cervera, Font, Raschi, Meroni. "Infectious origin of the antiphospholipid syndrome." Annals of the Rheumatic Diseases. 2006:65, 2-6.

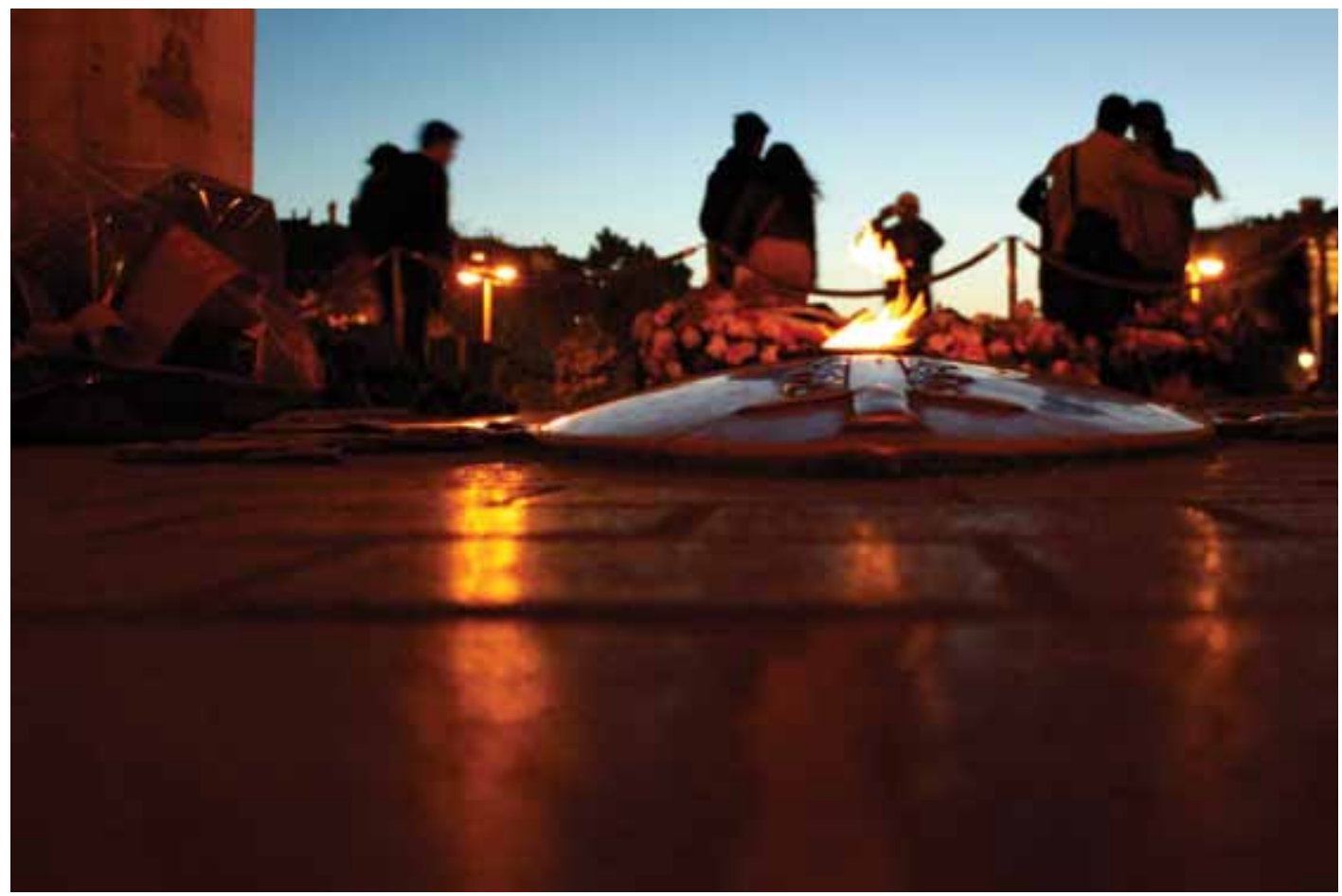

"Tomb"

Photograph by Cecilia Kelly, MD 